\title{
PASSIVE IMAGING EXPLOITING MULTIPLE SCATTERING USING DISTRIBUTED APERTURES
}

\author{
Ling Wang ${ }^{1}$, Il-Young Son ${ }^{2}$ and Birsen Yazıcl ${ }^{2 * *}$ \\ ${ }^{1}$ College of Information Science and Technology, \\ Nanjing University of Aeronautics and Astronautics, Nanjing,210016 China \\ ${ }^{2}$ Department of Electrical, Computer and Systems Engineering, \\ Rensselaer Polytechnic Institute, 110 8th Street, Troy, NY 12180 USA \\ E-mail: yazici@ecse.rpi.edu
}

\begin{abstract}
We develop a new passive image formation method capable of exploiting information about multiple scattering in the environment using measurements from a sparse array of receivers that rely on illumination sources of opportunity. We use a physics-based approach to model wave propagation and develop a statistical model that relates measurements at a given receiver to measurements at other receivers. We formulate the imaging problem as a spatially resolved binary hypothesis testing problem using the model between the measurements at different receivers, statistics of the objects to be imaged and statistics of the additive noise and clutter. We address the spatially resolved hypothesis testing problem by constraining the associated discriminant functional to be linear and by maximizing the signal-to-noise-ratio of the test-statistic, and use the resulting spatially resolved test-statistics to form the image. We present numerical simulations to demonstrate the performance of the passive imaging algorithm.
\end{abstract}

Index Terms - Passive imaging, multiple scattering, distributed apertures, binary hypothesis testing

\section{INTRODUCTION}

Passive imaging is performed by using a single or multiple receivers measuring the scattered wave field from an object to be imaged due to illumination sources of opportunity. Passive imaging covers a wide range of applications including acoustic, seismic and radar imaging.

Passive radar imaging is of particular interest in urban areas due to increasing number of broadcasting stations, mobile phone base stations, communication and navigation satellites. However, single-scattering assumption, on which classical received signal processing methods are based on, is not valid in urban areas. In this paper, we develop a passive

This work was supported by the Air Force Office of Scientific Research (AFOSR) under the agreement FA9550-04-1-0223 and FA9550-07-1-0363.

** Corresponding author image formation method capable of exploiting information about multiple scattering in the environment using measurements from a sparse array of receivers that rely on illumination sources of opportunity. The array of receivers can be distributed spatially in an arbitrary fashion with several hundred wavelengths apart. Such an array is referred to as a distributed aperture [1].

A number of passive imaging or detection approaches have been presented in the literature [2-13]. Our method has the following advantages as compared to the existing passive imaging techniques: 1) We derive a data model suitable for passive distributed sparse arrays which relates the statistics of measurements at a given receiver to the statistics of the measurements at other receivers, as well as the Green's function of the background environment. This model allows us to exploit multiple-scattering, as well as the a priori noise, clutter and target statistics. With the exception of [4] and [3], existing passive imaging techniques rely on the single-scattering assumption. 2) Our imaging method is formulated in an estimation-theoretic framework, specifically as a Generalized Likelihood Ratio Test (GLRT) [14]. We formulate the imaging problem as a binary hypothesis test with unknown target location. The GLRT is a particularly suitable framework for the sparse arrays due to limited aperture available. 3) Our approach does not necessarily require receivers with high directivity. 4) Our approach can be used in the presence of both cooperative and noncooperative sources of opportunity.

\section{A PASSIVE MEASUREMENT MODEL FOR DISTRIBUTED APERTURES}

We reserve $\mathbf{x}$ to denote location in 3D Euclidean space and $\boldsymbol{x}$ to denote location in 2D space. We denote operators $(\mathcal{G}, \mathcal{R}$, etc. $)$ with calligraphic letters. For a function $f, \hat{f}$ denotes its Fourier transform and $f^{*}$ denotes its complex conjugate. Bold font denotes vector quantities. Non-bold italic font denotes scalar quantities.

The propagation of electromagnetic waves in a medium 
due to distribution of sources can be adequately described by the scalar wave equation:

$$
\left(\nabla^{2}-\frac{1}{c^{2}} \partial_{t}^{2}\right) E(\mathbf{x}, t)=s(\mathbf{x}, t)
$$

where $c$ is the speed of electromagnetic waves in the medium, which can be expressed in terms of the background propagation speed, $c_{b}(\mathbf{x})$, and the perturbation due to deviation from the background reflectivity, $V(\mathbf{x})$ as $c^{2}(\mathbf{x})=\left(1 / c_{b}^{2}(\mathbf{x})+\right.$ $V(\mathbf{x}))^{-1}, E(\mathbf{x}, t)$ is the electric field and $s(\mathbf{x}, t)$ is the source term, at location $\mathbf{x} \in \mathbb{R}^{3}$ and time $t \in \mathbb{R}^{+}$. For an isotropic point source located at $\mathbf{x}_{0}, s(\mathbf{x}, t)=p(t) \delta\left(\mathbf{x}-\mathbf{x}_{0}\right)$ where $p(t)$ is the transmitted waveform. The propagation medium is characterized by the Green's function satisfying

$$
\left(\nabla^{2}-\frac{1}{c^{2}} \partial_{t}^{2}\right) g(\mathbf{x}, \mathbf{y}, t)=\delta(\mathbf{x}-\mathbf{y}) \delta(t) .
$$

For typical carrier frequencies used in surveillance radar the incident field decays rapidly as it penetrates the ground. We can then write $V(\mathbf{x})$ in terms of a 2-D function, as in $V(\mathbf{x})=V(\boldsymbol{x}) \delta\left(x_{3}-h(\boldsymbol{x})\right)$ where $\mathbf{x}=\left(\boldsymbol{x}, x_{3}\right), \boldsymbol{x} \in \mathbb{R}^{2}$ and $h: \mathbb{R}^{2} \rightarrow \mathbb{R}$ represents the known ground topography.

Since it is not reasonable to expect that the background model can account for arbitrarily fine details of the background, the reflectivity function $V$ could be decomposed into two parts, $V=T+C$ where $T$ denotes the objects of interest called target and $C$ denotes heterogeneities that are neither target nor a part of the background medium, called clutter.

Let $E$ denote the total field in the medium. Then the scattered field measurements at the receiver located at $\mathbf{x}_{0}$ can be modeled as

$$
m(t)=\int g\left(\mathbf{x}_{0}, \boldsymbol{y}, t-\tau\right) T(\boldsymbol{y}) \partial_{\tau}^{2} E(\boldsymbol{y}, \tau) d \boldsymbol{y} d \tau+\tilde{n}(t)
$$

where

$$
\tilde{n}(t)=\int g\left(\mathbf{x}_{0}, \boldsymbol{y}, t-\tau\right) C(\boldsymbol{y}) \partial_{\tau}^{2} E(\boldsymbol{y}, \tau) d \boldsymbol{y} d \tau+n(t) .
$$

we define $g(\mathbf{x}, \boldsymbol{y}, t)$ as the 3D Green's function equal to $g(\mathbf{x},(\boldsymbol{y}, h(\boldsymbol{y})), t), \boldsymbol{y}=\left(y_{1}, y_{2}\right)$. The first integral term in (4) represents the measurement due to clutter. We denote it by $n_{C}(t)$. Without loss of generality, we assume that $n_{C}(t)$ has zero-mean with finite variance and autocovariance function. $n(t)$ denotes the additive thermal noise at the receiver. we assume that $n(t)$ is white with variance $\sigma_{n}^{2}$ and is uncorrelated with $n_{C}(t)$. Note that (3) can be linearized by the first Born approximation, after which the total field on the right side of (3) is replaced by the incident field.

In Fourier domain, (3) becomes

$$
\hat{m}(\omega)=\int \hat{g}\left(\mathbf{x}_{0}, \boldsymbol{y}, \omega\right) T(\boldsymbol{y}) \omega^{2} \hat{E}(\boldsymbol{y}, \omega) d \boldsymbol{y}+\hat{\tilde{n}}(\omega) .
$$

For passive detection and imaging applications, the incident field is not known, since the information on the transmitted waveforms and the location of the transmitters may not be available. Here, we develop an alternative measurement model that expresses measurements at each receiver in terms of the measurements at a different receiver. The model involves a back-propagation operation and a forwardpropagation operation.

The forward-propagation operator is an integral operator that maps the total field and the target at a hypothetical target location $\boldsymbol{y}$ to the scattered field at the $i^{\text {th }}$ receiver, i.e.,

$$
\left[\mathcal{G}_{\boldsymbol{y}, i} u\right](\omega)=\int W_{s}\left(\boldsymbol{y}^{\prime}, \boldsymbol{y}\right) \hat{g}\left(\mathbf{x}_{i}, \boldsymbol{y}^{\prime}, \omega\right) u\left(\boldsymbol{y}^{\prime}, \omega\right) d \boldsymbol{y}^{\prime}
$$

where $u(\boldsymbol{y}, \omega)=T(\boldsymbol{y}) \omega^{2} \hat{E}(\boldsymbol{y}, \omega)$ and $W_{s}\left(\boldsymbol{y}^{\prime}, \boldsymbol{y}\right)$ is a spatial windowing function of unit amplitude centered at a hypothetical target location $\boldsymbol{y}$. Note that the forward-propagation operator is $\boldsymbol{y}$ dependent due to the spatial windowing function $W_{s}$. We define the back-propagation operator as the inverse of $\mathcal{G}_{\boldsymbol{y}, i}$, denote it with $\mathcal{G}_{\boldsymbol{y}, i}^{-1}$. Since the operator $\mathcal{G}_{\boldsymbol{y}, i}$ is applied only at a single spatial location $\mathbf{x}_{i}$, its inverse may not exist for all locations $\boldsymbol{y}^{\prime} \in D_{W_{s}(\boldsymbol{y})} \cdot \mathcal{G}_{\boldsymbol{y}, i}^{-1}$ may be replaced with its pseudoinverse.

In an ideal scenario, where there is no noise or clutter in the measurements, we can express the $i^{\text {th }}$ measurement in terms of the $j^{\text {th }}$ measurement as follows:

$$
\hat{m}_{i}^{j(0)}(\omega)=\mathcal{G}_{\boldsymbol{y}, i} \mathcal{G}_{\boldsymbol{y}, j}^{-1} \hat{m}_{j}^{(0)}(\omega)
$$

where $\hat{m}_{j}^{(0)}(\omega)$ and $\hat{m}_{i}^{j(0)}$ are the noise and clutter-free measurements at the $j^{\text {th }}$ and $i^{\text {th }}$ receivers, respectively. In the presence of noise and clutter, $\hat{\tilde{n}}_{j}$ is also back-propagated along with $\hat{m}_{j}^{(0)}(\omega)$. Therefore, if we denote $\hat{m}_{j}=\hat{m}_{j}^{(0)}+\hat{\tilde{n}}_{j}$, the full expression for an alternative measurement model at the $i^{\text {th }}$ receiver in terms of the $j^{\text {th }}$ measurement, i.e., $\hat{m}_{i}^{j}(\omega)$, becomes

$$
\begin{aligned}
\hat{m}_{i}^{j}(\omega) & =\mathcal{G}_{\boldsymbol{y}, i} \mathcal{G}_{\boldsymbol{y}, j}^{-1} \hat{m}_{j}^{(0)}(\omega)+\mathcal{G}_{\boldsymbol{y}, i} \mathcal{G}_{\boldsymbol{y}, j}^{-1} \hat{\tilde{n}}_{j}(\omega)+\hat{\tilde{n}}_{i}(\omega) \\
& =\mathcal{G}_{\boldsymbol{y}, i} \mathcal{G}_{\boldsymbol{y}, j}^{-1} \hat{m}_{j}(\omega)+\hat{\tilde{n}}_{i}(\omega) .
\end{aligned}
$$

Suppose there are $N$ receive antennas located at $\mathbf{x}_{1}, \ldots \mathbf{x}_{N}$, indexed by $1, \ldots, N$. A vector measurement model can be formed by taking one of the receivers as a reference. Without loss of generality, we take the $j^{\text {th }}$ receiver as a reference and form the following measurement vector,

$$
\mathbf{m}=\left[\begin{array}{llll}
\hat{m}_{1}^{j} & \hat{m}_{2}^{j} & \cdots & \hat{m}_{N}^{j}
\end{array}\right]^{T}
$$

where $\hat{m}_{i}^{j}, i=1, \cdots, N$ and $i \neq j$, denotes the measurement $\hat{m}_{i}$ modeled in terms of the reference measurement $\hat{m}_{j}$. Similarly, we can vectorize the "reference measurements", $\hat{m}_{j}$ 's, and the measurements due to noise and clutter:

$$
\begin{aligned}
\mathbf{m}_{\mathbf{r}} & =\left[\begin{array}{llll}
\hat{m}_{j} & \hat{m}_{j} & \cdots & \hat{m}_{j}
\end{array}\right]^{T} \\
\mathbf{n} & =\left[\begin{array}{llll}
\hat{\tilde{n}}_{1} & \hat{\tilde{n}}_{2} & \cdots & \hat{\tilde{n}}_{N}
\end{array}\right]^{T}
\end{aligned}
$$

where in $\hat{\tilde{n}}_{i}, i \neq j$ as defined in (4) and (5). Note that $\mathbf{m}, \mathbf{m}_{\mathbf{r}}$ and $\mathbf{n}$ are all vectors of length $(N-1)$. 
The composition of the back-propagation and forwardpropagation operators can be represented as a diagonal matrix given by

$$
\mathbf{G}_{\boldsymbol{y}}=\operatorname{diag}\left[\begin{array}{llll}
\mathcal{G}_{\boldsymbol{y}, 1} \mathcal{G}_{\boldsymbol{y}, j}^{-1} & \mathcal{G}_{\boldsymbol{y}, 2} \mathcal{G}_{\boldsymbol{y}, j}^{-1} & \cdots & \mathcal{G}_{\boldsymbol{y}, N} \mathcal{G}_{\boldsymbol{y}, j}^{-1}
\end{array}\right]
$$

where $i \neq j$ and $\mathbf{G}_{\boldsymbol{y}}$ is $(N-1) \times(N-1)$.

Using (8), (9)-(12), we form the vectorized passive measurement model as follows:

$$
\mathbf{m}(\omega)=\mathbf{G}_{\boldsymbol{y}} \mathbf{m}_{\mathbf{r}}(\omega)+\mathbf{n}(\omega)
$$

for some range of $\omega$. Note that in (13), all the operations are understood to be elementwise.

\section{PASSIVE IMAGING AS A SPATIALLY RESOLVED BINARY HYPOTHESIS TESTING PROBLEM}

We formulate the imaging problem as a binary hypothesis testing problem which has its roots in the Generalized Likelihood Ratio Test (GLRT) [14]. The application of the GLRT formalism to imaging involves modeling of the scene as a collection of point targets with unknown locations and setting up a spatially resolved hypothesis testing problem to evaluate the presence or absence of a target. The image is formed by the spatially resolved test-statistic where the location and possibly the shapes of the targets can be identified by thresholding the image. Here, we determine the test-statistic by maximizing the SNR of the test-statistic while constraining the associated discriminant functional to be linear.

The spatially resolved binary hypothesis test can be expressed as:

$$
\begin{aligned}
& \mathcal{H}_{0}: \mathbf{m}=\mathbf{n} \quad \Rightarrow \text { target absent } \\
& \mathcal{H}_{1}: \mathbf{m}=\mathbf{G}_{\boldsymbol{y}} \mathbf{m}_{\mathbf{r}}+\mathbf{n} \quad \Rightarrow \text { target present }
\end{aligned}
$$

where $\mathbf{G}_{\boldsymbol{y}}, \mathbf{m}_{r}, \mathbf{m}$ and $\mathbf{n}$ are as defined in (9)-(13).

Using (7), (10), (11) and (14), we obtain

$$
\begin{aligned}
\mathrm{E}\left[\mathbf{m} \mid \mathcal{H}_{0}\right] & =\mathbf{0} \\
\operatorname{Cov}\left[\mathbf{m} \mid \mathcal{H}_{0}\right] & =\mathbf{R}_{\mathbf{n}}=: \mathbf{R}_{0} \\
\mathrm{E}\left[\mathbf{m} \mid \mathcal{H}_{1}\right] & =\mathbf{G}_{\boldsymbol{y}} \mathrm{E}\left[\mathbf{m}_{\mathbf{r}} \mid \mathcal{H}_{1}\right]=\mathbf{G}_{\boldsymbol{y}} \overline{\mathbf{m}}_{\mathbf{r}} \\
\operatorname{Cov}\left[\mathbf{m} \mid \mathcal{H}_{1}\right] & =\mathbf{G}_{\boldsymbol{y}}\left(\mathbf{R}_{T}+\mathbf{R}_{\mathbf{n}}\right) \mathbf{G}_{\boldsymbol{y}}^{H}+\mathbf{R}_{\mathbf{n}}=: \mathbf{R}_{1}
\end{aligned}
$$

where $\overline{\mathbf{m}}_{\mathbf{r}}$ denotes $\mathrm{E}\left[\mathbf{m}_{\mathbf{r}} \mid \mathcal{H}_{1}\right], \mathbf{G}_{\boldsymbol{y}}^{H}$ denotes the Hermitian transpose of $\mathbf{G}_{\boldsymbol{y}}, \mathbf{R}_{T}$ denotes the autocovariance of the noiseand clutter- free reference measurements in the presence of a statistical target and $\mathbf{R}_{\mathbf{n}}$ denotes the autocovariance of the measurements due to clutter and noise, i.e., $\mathbf{R}_{\mathbf{n}}\left(\omega, \omega^{\prime}\right)=$ $\mathrm{E}\left[\mathbf{n}(\omega) \mathbf{n}^{H}\left(\omega^{\prime}\right)\right]$.

The linear discriminant functional involved in our problem has the form

$$
\lambda=\langle\mathbf{m}, \mathbf{w}\rangle:=\int \mathbf{w}^{H} \mathbf{m} d \omega=\sum_{i \neq j} \int w_{i}^{j *}(\omega) \hat{m}_{i}^{j}(\omega) d \omega
$$

where $\lambda$ denote the the output of the discriminant functional, which we call the test-statistic and $\mathbf{w}$ is a template given by $\mathbf{w}=\left[\begin{array}{ll}w_{1}^{j} & w_{2}^{j} \cdots w_{N}^{j}\end{array}\right]^{T}$. We determine the template by maximizing the signal-to-noise ratio (SNR) of $\lambda$ [14]. The resulting optimal linear template is

$$
\mathrm{w}^{\star}=\overline{\mathcal{R}}^{-1} \mathbf{G}_{\boldsymbol{y}} \overline{\mathbf{m}}_{\mathbf{r}}
$$

where $\mathcal{R}$ is a non-negative definite symmetric operator with the matrix kernel $1 / 2\left(\mathbf{R}_{1}+\mathbf{R}_{0}\right)$.

(20) shows that the optimal template is location dependent. For deterministic targets, under the assumption that the measurements due to clutter and noise are wide sense stationary and mutually uncorrelated, (20) becomes

$$
\mathbf{w}^{\star}=\overline{\mathbf{S}}^{-1}(\omega) \mathbf{G}_{\boldsymbol{y}}(\omega) \overline{\mathbf{m}}_{\mathbf{r}}(\omega) .
$$

where $\overline{\mathbf{S}}$ is a diagonal matrix with elements $\bar{S}_{i}(\omega), i=$ $1, \cdots, N$ and $i \neq j$, which is a function of the power spectral density function of the measurements at the $i^{\text {th }}$ receiver due to clutter and noise and the kernel of $\mathbf{G}_{\boldsymbol{y}}$.

\section{NUMERICAL SIMULATIONS}

We conducted numerical simulations to verify the our imaging theory using a point target.

We assumed that the transmitted signal is continuouswave (CW) with $900 \mathrm{MHz}$ carrier frequency and $10 \mathrm{~ns}$ duration. The sampling rate of the signal was chosen to be $5 \mathrm{GHz}$. We assumed that all of transmitters are transmitting the same waveform.

We consider a scene of size $[0,70] \times[0,100] \mathrm{m}^{2}$ with flat topography. The point target was assumed to be at $\left[\begin{array}{lll}45.34 & 45 & 1\end{array}\right]^{T} \mathrm{~m}$ with unit reflectivity. Both the receivers and transmitters were assumed to be located on the same $z$ plane, $z=6$, and lie on a straight line, equidistant from each other. The three receivers were located at $\left[\begin{array}{lll}58.8 & 0 & 6\end{array}\right]^{T}$, $\left[\begin{array}{lll}28.3 & 0 & 6\end{array}\right]^{T}$ and $\left[\begin{array}{lll}-2.1 & 0 & 6\end{array}\right]^{T}$, all in meters. The transmitter was located at $\left[\begin{array}{lll}28.3 & 70 & 6\end{array}\right]^{T} \mathrm{~m}$ for the singletransmitter case, and $\left[\begin{array}{lll}36.6 & 0 & 6\end{array}\right]^{T} \mathrm{~m}$ and $\left[\begin{array}{lll}20.1 & 0 & 6\end{array}\right]^{T} \mathrm{~m}$ for the two-transmitter case.

For the multiple scattering environment, we used a "shoot-and-bounce", multi-path propagation model and considered a specular reflecting wall located at $x=0$.

In all the experiments, the thermal noise is simulated as the additive white Gaussian process.

Fig. 1 and Fig. 2 show the reconstructed images for the point target model in free-space and multi-path environments, respectively. Comparing Fig. 1(b) with Fig. 1(a), and Fig. 2(b) with Fig. 2(a), we see that in both free-space and multi-path propagation environments, the strength of the image at the target location increases with the increasing number of transmitters. However, multiple transmitters also induce artifacts in the images due to perfect cross-correlation between the transmitted waveforms as predicted by the theory. 


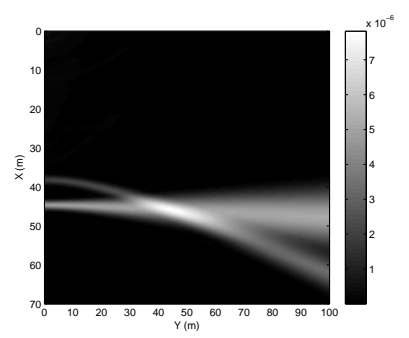

(a)

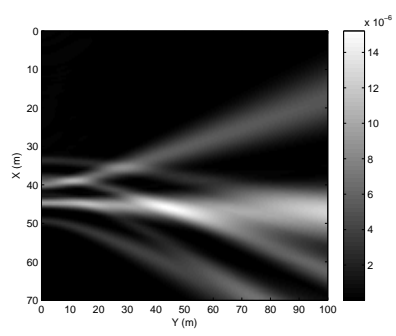

(b)
Fig. 1. The reconstructed images of a point target in freespace in the presence of 3 receivers and (a) a single transmitter, and (b) 2 transmitters.

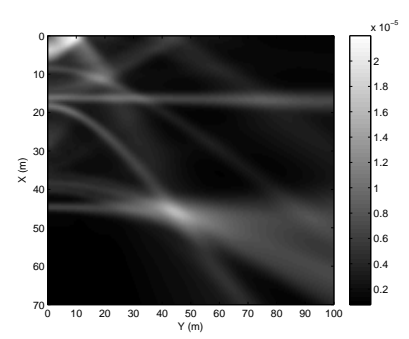

(a)

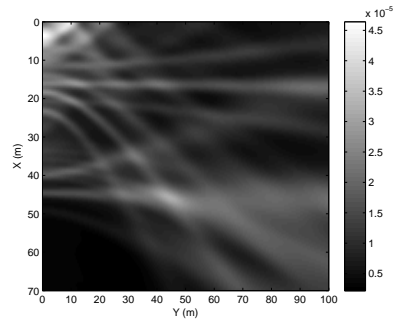

(b)
Fig. 2. The reconstructed images of a point target in multipath propagation environments in the presence of 3 receivers and (a) a single transmitter, and (b) 2 transmitters.

Note that looking at Fig. 2, we see that the strength of the target increased by almost an order-of-magnitude when we exploited the multi-path effect as compared to the image reconstructed in free-space propagation environment shown in Fig. 1. However, there are also additional artifacts.

\section{CONCLUSION}

In this work, we presented a new passive image formation method using sparse distributed apertures, which is capable of operating in both free-space and multiple-scattering environments. A detailed resolution analysis of the imaging method will be presented in another paper. Finally, we note that this imaging method is not limited to radar, and can easily be adapted to similar passive imaging problems in acoustics, geophysics or microwave imaging.

\section{REFERENCES}

[1] R. Adve, R. Schneibler, G. Genello, and P. Antonik, "Waveform-space-time adaptive processing for distributed aperture radars," pp. 93-97, 2005.

[2] H. D. Griffiths and C. J. Baker, "Passive coherent location radar systems. part 1: Performance prediction," IEE
Proceedings of Radar, Sonar and Navigation, vol. 152, no. 3, pp. 153-159, June 2005.

[3] L. Borcea, G. Papanicolaou, and C. Tsogka, "Interferometric array imaging in clutter," Inverse Problems, vol. 21, pp. 1419-1460, 2005.

[4] L. Borcea, G. Papanicolaou, and C. Tsogka, "Coherent interferometric imaging in clutter," Geophysics, vol. 71, no. 4, pp. SI165-SI175, July 2006.

[5] C. J. Baker, H. D. Griffiths, and I. Papoutsis, "Passive coherent location radar systems. part 2: Waveform properties," IEE Proceedings of Radar, Sonar, and Navigation, vol. 152, no. 3, pp. 160-168, June 2005.

[6] P. E. Howland, D. Maksimiuk, and G. Reitsma, "Fm radio based bistatic radar," IEE Proceedings of Radar, Sonar and Navigation, vol. 152, no. 3, pp. 107-115, June 2005.

[7] D. K. P. Tan, H. Sun, Y. Lu, M. Lesturgie, and H. L. Chan, "Passive radar using global system for mobile communication signal: theory, implementation and measurements," IEE Proceedings of Radar, Sonar, and Navigation, vol. 152, no. 3, pp. 116-123, June 2005.

[8] V. Koch and R. Westphal, "New approach to a multistatic passive radar sensor for air/space defense," IEEE Aero. Electron. Syst. Mag., vol. 10, pp. 24-32, Nov. 1995.

[9] D. Poullin, "Passive detection using digital broadcasters (dab, dvb) with cofdm modulation," IEE Proceedings of Radar, Sonar and Navigation, vol. 152, no. 3, pp. 143152, June 2005.

[10] X. He, M. Cherniakov, and T. Zeng, "Signal detectability in ss-bsar with gnss non-cooperative transmitter," IEE Proceedings of Radar, Sonar and Navigation, vol. 152, no. 3, pp. 124-132, June 2005.

[11] K. S. Kulpa, "Multi-static entirely passive detection of moving targets and its limitations," IEE Proceedings of Radar, Sonar, and Navigation, vol. 152, no. 3, pp. 169173, June 2005.

[12] S. J. Norton, B. J. Carr, and A. J. Witten, "Passive imaging of underground acoustic sources," The Journal of the Acoustical Society of America, vol. 119, no. 5, pp. 2840-2847, May 2006.

[13] C. E. Yarman and B. Yazıcı, "Synthetic aperture hitchhiker imaging," IEEE Transactions on Imaging Processing, vol. 17, no. 11, pp. 2156-2173, November 2008.

[14] S. M. Kay, Fundamentals of Statistical Signal Processing, Vol. I and Vol. II, Prentice Hall, 1998. 